\title{
Haploidentical versus HLA-matched sibling transplantation for refractory acute leukemia undergoing sequential intensified conditioning followed by DLI: an analysis from two prospective data
}

Sijian $\mathrm{Yu}^{1,9+}$, Fen Huang ${ }^{1+}$, Zhiping Fan ${ }^{1 \dagger}, \mathrm{Li}_{\mathrm{X}} \mathrm{Xuan}{ }^{1}$, Danian $\mathrm{Nie}^{3}$, Yajing $\mathrm{Xu}^{4}$, Ting Yang ${ }^{5}$, Shunqing Wang ${ }^{6}$,

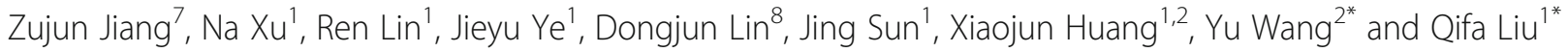

\begin{abstract}
Background: Compared with HLA-matched sibling donor (MSD) transplant, the outcomes of haploidentical donor (HID) transplant for refractory acute leukemia need to be further explored. In this study, we compared the outcomes of HID with MSD for refractory acute leukemia.

Patients and methods: This study population came from two prospective multicenter trials (NCT01883180, NCT02673008). Two hundred and seventy-eight patients with refractory acute leukemia were enrolled in this study, including 119 in HID group and 132 in MSD group. Sequential intensified conditioning was employed in all patients, and donor lymphocyte infusion (DLI) was administered in patients in the absence of active GVHD and according to minimal residual disease (MRD) from day +60 post-transplantation for preventing relapse.
\end{abstract}

Results: The complete remission of leukemia by day +30 post-transplant were $94 \%$ and $93 \%$, respectively, in HID and MSD groups $(p=.802)$. The 1-year incidence of grades $\|-I V$ acute GVHD was $62 \%$ and $54 \%(p=.025)$, and 3 -year incidence of chronic GVHD was 55\% and 55\% ( $p=.789)$, respectively, in two groups. HID transplant had lower incidence of first episode of MRD positivity and relapse than MSD transplant ( $28 \%$ vs $45 \%, p=.006 ; 26 \%$ vs $38 \%, p=.034$ ). There was higher infectionrelated mortality in HID than MSD ( $8 \%$ vs $2 \%, p=.049$ ) within the first 100 days' post-transplant. The 5 -year overall survival was $46 \%$ and $42 \%(p=.832)$, respectively; the 5 -year disease-free survival was $43 \%$ and $39 \%(p=.665)$, in HID and MSD groups, respectively.

Conclusions: HID transplant has lower relapse, but higher infection-related mortality and similar survival rates in refractory acute leukemia by the strategy of sequential intensified conditioning followed by DLI compared with MSD transplant.

Keywords: HLA-matched sibling, Haploidentical, Refractory acute leukemia, Similar survival, Transplantation

\footnotetext{
* Correspondence: ywyw3172@sina.com; liuqifa628@163.com

${ }^{\dagger}$ Sijian Yu, Fen Huang and Zhiping Fan contributed equally to this work.

2Department of Hematology, Peking University People's Hospital, Peking

University Institute of Hematology, No.11 South Street of Xizhimen, Xicheng

District, Beijing 100044, China

'Department of Hematology, Nanfang Hospital, Southern Medical University,

No.1838 North Guangzhou Avenue, Guangzhou 510515, China

Full list of author information is available at the end of the article
}

C C The Author(s). 2020 Open Access This article is licensed under a Creative Commons Attribution 4.0 International License, which permits use, sharing, adaptation, distribution and reproduction in any medium or format, as long as you give appropriate credit to the original author(s) and the source, provide a link to the Creative Commons licence, and indicate if changes were made. The images or other third party material in this article are included in the article's Creative Commons licence, unless indicated otherwise in a credit line to the material. If material is not included in the article's Creative Commons licence and your intended use is not permitted by statutory regulation or exceeds the permitted use, you will need to obtain permission directly from the copyright holder. To view a copy of this licence, visit http://creativecommons.org/licenses/by/4.0/ The Creative Commons Public Domain Dedication waiver (http://creativecommons.org/publicdomain/zero/1.0/) applies to the data made available in this article, unless otherwise stated in a credit line to the data. 


\section{Introduction}

So far, allogeneic hematopoietic stem cell transplantatio$\mathrm{n}$ (allo-HSCT)remains the most effective way to cure refractory leukemia [1-4] and undergoing allo-HSCT promptly is essential for them. Recently, some studies showed that intensified conditioning followed by allo-HSCT could achieve acceptable outcomes for refractory leukemia [5-9]. In our transplant center, a strategy of sequential intensified conditioning followed by donor lymphocyte infusion (DLI) was implemented since 2009, and encouraging outcomes were reported previously [10].

Quick access to appropriate donors is one of the key elements to the success of transplantation for refractory leukemia. As it is known, only 25-30\% patients can get a HLA-matched sibling donor (MSD), and most patients cannot wait to search for a suitably matched unrelated donor if it is not already available. With improvements having been made in haploidentical donor (HID) transplant strategies, some studies showed that transplant outcomes of HID were similar to MSD in acute leukemia [11-14], but data comparing HID with MSD for refractory leukemia are quite limited $[15,16]$. A recent retrospective analysis from EBMT [16] showed that HID transplant was associated with inferior survival rates and higher non-relapse mortality (NRM). However, the study population was very heterogeneous in terms of conditioning regimen, graft versus host disease (GVHD) prophylaxis, intervention strategy posttransplantation, and so on. Besides, some studies found a lower relapse incidence (RI) in HID than MSD transplant for high risk leukemia, suggesting superior graft versus leukemia (GVL) in HID [17-19].

In this study, we analyzed data from two prospective multicenter trials, to investigate the transplant outcomes of HID versus MSD for refractory acute leukemia by using our transplant strategy of sequential intensified conditioning followed by DLI [10].

\section{Patients and methods}

This study population came from two prospective multicenter trials (NCT01883180, NCT02673008). Patients undergoing allo-HSCT between June 2013 and December 2017 were enrolled in this study if they met the following criteria: (1) refractory acute leukemia, (2) no complete remission (no-CR) at transplant, (3) using HID or MSD as donors, and (4) first alloHSCT. Patients with FLT3-ITD and BCR/ABL were excluded because they received sorafenib or tyrosine kinase inhibitors post-transplant. The study was performed in accordance with the modified Helsinki Declaration, and the protocol was approved by our ethical review boards before study initiation. Informed consent was obtained from the donors and recipients.

\section{Conditioning and transplants}

The sequential intensified conditioning regimen was used in all patients, including fludarabine (Flu) $30 \mathrm{mg} / \mathrm{m}^{2} /$ day and cytarabine (Ara-C) $2 \mathrm{~g} / \mathrm{m}^{2} /$ day from days -10 to -6 , 4.5 Gy of total body irradiation (TBI) on days -5 and -4 , cyclophosphamide (CY) $60 \mathrm{mg} / \mathrm{kg} /$ day, and etoposide (VP16) $15 \mathrm{mg} / \mathrm{kg} /$ day on days -3 and - 2 [7]. As for donor selection, MSD (6/6 matching HLA-A, B, and DR loci) was the first choice. If MSD and a suitably matched unrelated donor (> 8 of 10 matching HLA-A, B,C,DR, and DQ loci) were unavailable, patients would be transplanted with HID [4].HID patients were transplanted with a combination of bone marrow (BM) and peripheral blood stem cell (PBSC) grafts, whereas MSD patients received PBSC grafts. Ciclosporin A (CsA) + methotrexate (MTX) were administered in patients undergoing MSD transplants for GVHD prophylaxis. CsA + MTX + antithymocyte globulin (ATG) and mycophenolate were used in patients receiving HID [10].The total dose of ATG (rabbit antihuman thymocyte immunoglobulin, ImtixSangstat, Lyon, France) was randomly assigned as $7.5 \mathrm{mg} / \mathrm{kg}$ from days 3 to -1 or $10.0 \mathrm{mg} / \mathrm{kg}$ from days -4 to -1 in one trial (NCT01883180), and just $10.0 \mathrm{mg} / \mathrm{kg}$ from days -4 to -1 for all patients in another trial (NCT02673008).

\section{Surveillance and intervention for relapse}

BM samples analyzed at 1, 2, 3, 4, 6, 8, 10, and 12 months within 1 year post-transplantation, then at 3-month intervals from the 13th to 24th month, and 4-month intervals from the 25th to 36th month for the monitoring of morphology and minimal residual disease (MRD). If MRD was positive, the test was repeated in 1 week. Eight-color multiparameter flow cytometry (MFC) and quantitative PCR (qPCR) were used for the detection of MRD as previously described [20-22].For MFC method, positive MRD was considered when a cluster of more than 25 cells with leukemia-associated immunophenotypes (LAIP) and SSC characteristics identified in all plots of interest and carrying at least two LAIP markers identified at diagnosis that was observed. For those without LAIP markers at diagnosis, MRD was identified as a cell population showing deviation from the normal patterns of antigen expression seen on specific cell lineages at specific stages of maturation compared with either normal or regenerating marrow. A lower limit of detection of $0.01 \%$ was targeted. When abnormal cells were identified, the cells were quantified as a percentage of the total CD45 white cell events. Any measurable level of MRD was considered positive. Also, leukemiarelated specific genes, including NPM1, RUNX1RUNX1T1, and CBF $\beta / M Y H 11$ were detected by $\mathrm{qPCR}$ in AML. The cutoff value was $0.001 \%$. Subjects were defined as MRD+ if they had 2 consecutive positive results using FCM or PCR or were both positive in a single sample.

The prevention of leukemic relapse included early tapering of immunosuppressant, prophylactic, and preemptive therapies according to our previous literatures [10, 22]. 
CsA was withdrawn by $10 \%$ /week in patients without acute GVHD (aGVHD) by day + 30 post-transplantation. Prophylactic DLI was given in patients once on day +90 post-transplantation when donor lymphocytes were available without active aGVHD [10]. Preemptive DLI was conducted in patients with MRD+ post-transplants (post$\mathrm{MRD}+$ ) without active GVHD from day +60 , which was given monthly until GVHD occurred or MRD became negative or for a total of 4 times. Donor lymphocytes were obtained from G-CSF mobilized peripheral blood [10, 23]. The $\mathrm{CD}^{+} \mathrm{T}$ cells count for per DLI (pDLI) was $3 \times 10^{7} /$ $\mathrm{kg}$. Short-term immunosuppressive agents were used for prevention of GVHD after DLI [23]. For post-MRD+ AML patients with active GVHD, preemptive decitabine with a dose of $20 \mathrm{mg} / \mathrm{m}^{2} /$ day for 5 consecutive days was administered monthly until MRD turned negative or hematological relapse or for a total of 4 times.

\section{Infection prophylaxis}

Infection prophylaxis was performed as previously described [24, 25]. Oral sulfamethoxazole and norfloxacin were used in all cases. The EBV- and CMV-DNA loads in the blood were measured regularly by real-time qPCR. EBV-DNA or CMV-DNA was considered positive when the copies exceeded 500 copies $/ \mathrm{ml}$. Preemptive therapy was given to the patients with EBV or CMV-DNA-emia [25]. Antifungal agents were administered 5 days before transplantation. Oral fluconazole $(0.3 \mathrm{~g} /$ day $)$ was used for up to + 60 days post-transplantation in patients with no history of invasive fungal infection (IFI); for patients with a history of IFI, antifungal agents for secondary prophylaxis based on response to initial antifungal therapy were used for up to + 90 days post-transplantation [26].

\section{Evaluation endpoints and definitions}

The primary endpoint included RI and overall survival (OS). Secondary endpoints included engraftment, disease response, GVHD, infections, NRM, disease-free survival (DFS), GVHD-free, and relapse-free survival (GRFS). Assessments of engraftment and chimerism were previously described in detail [7]. Relapse was defined by morphologic evidence in the peripheral blood, marrow, or extramedullary sites. On days 0 and +30 post- transplantation, disease response was assessed by $\mathrm{BM}$ aspiration. $\mathrm{CR}$ was defined as $<5 \%$ blasts with no evidence of dysplasia in the BM and no manifestations of leukemia outside the hematopoietic system. Partial remission (PR) was defined as $5-20 \%$ blasts with or without extramedullary leukemia. Non-remission (NR) was defined as a failure to obtain CR. NRM was estimated as death without evidence of leukemia recurrence. DFS was defined as survival in continuous complete remission without relapse. GRFS events were defined as grades III-IV acute GVHD (aGVHD), chronic GVHD (cGVHD) requiring systemic immunosuppressive treatment, leukemia relapse, or death from any cause during follow-up after allo-HSCT [27]. aGVHD and cGVHD were graded according to the literatures $[28,29]$. CMV-associated disease was defined by the presence of clinical symptoms or signs of end organ disease, combined with the evidence of CMV infection in a tissue biopsy specimen. EBV-associated diseases were classified into EBV-associated post-transplant lymphoproliferative diseases and EBV-associated other diseases [25]. Acute leukemia, including acute myeloid leukemia (AML), acute lymphoblastic leukemia (ALL), and acute leukemia of ambiguous lineage (ALAL), was defined according to World Health Organization guideline [30]. Refractory acute leukemia was defined as primary induction failure (PIF) after two or more cycles of chemotherapy or relapse refractory to salvage chemotherapy $[7,16]$.Genetics, including cytogenetics and molecular genetics, was defined as favorable, intermediate, and poor-risk in acute myeloid leukemia (AML) [31] and favorable and poor-risk in acute lymphoblastic leukemia (ALL) according to the National Comprehensive Cancer Network guideline [32]. Prophylactic and preemptive therapies were defined as interventions for MRD- and MRD+ patients without hematologic relapse, respectively.

\section{Statistical analysis}

Analysis was performed on June 30, 2019. Variables related to patients, disease, and transplant characteristics between the two groups were compared using Fisher's exact test for categorical variables and Mann-Whitney $U$ tests for continuous variables. Numerical variables were analyzed as categories based on their values being below or above the median of the entire cohort. DFS, OS, and GRFS were calculated using the Kaplan-Meier method and compared by the log-rank test. Cumulative incidences were estimated for engraftment, GVHD, relapse, NRM, and infections to accommodate competing risks. Competing risk for engraftment was death without engraftment, competing risks for GVHD included death without GVHD and relapse, competing risks for infections included death without infections and relapse, relapse was a competing risk for NRM, and NRM was a competing risk for relapse. A cox proportional hazards model was used for analysis of risk factors for time-toevent variables. Fine and Gray model was used for analysis of endpoints involving competing risks [33].The following variables were included in the univariate analysis: donor type, gender, age, underlying diseases, genetics, white blood cell count at diagnosis, BM blasts pre/postconditioning, MRD post-transplant, DLI, aGVHD, and cGVHD. Only variables with $p<0.10$ were included in the multivariate analysis. $P$ values of less than 0.05 were considered statistically significant. The Stata SE 12.0 (StataCorp LP) and $\mathrm{R}$ version 3.4.3 ( $\mathrm{R}$ Development Core Team, Vienna, Austria) were used for all data analysis. 


\section{Results}

\section{Patients and transplant characteristics}

There were 251 patients enrolled in this study, including 119 in HID group and 132 in MSD group (Fig. 1). The median age was 29 (range 14-56) years. Primary diseases included AML $(n=111)$, ALL $(n=115)$, and ALAL $(n=25)$. Patient and transplant characteristics are shown in Table 1. There was no significant difference between two groups in terms of baseline factors in Table $1(p>.050)$.

\section{Engraftment and disease response}

Analyses of chimerism showed that 225 cases (91\%) achieved full donor chimerism and 23 (9\%) mixed chimerism by day +30 post-transplantation except for two patients who died of infection (1 in HID and 1 in MSD group) and one of primary graft failure in HID group. The median time of neutrophil recovery was 13 (range, 9-48) and 12 (range, 9-41) days in HID and MSD groups $(p=.096)$, respectively. The median time of platelet engraftment was 18 (range, 10-90) and 17 (range, 9-70) days, respectively, in two groups ( $p=.131$ ).

The count of BM blasts was analyzed on day 0 to testify disease response from sequential intensified conditioning. The median percentage of BM blasts decreased from 32\% (range, 7.0-98.0\%) pre-conditioning to 3.0\% (range, 0.0$19.0 \%$ ) on day 0 . The percentage of BM blasts preconditioning and on day 0 was similar between two groups ( $p=.602 ; p=.563$, respectively). On day 30 post-transplantation, $94 \%$ of the patients achieved $\mathrm{CR}$ and $6 \% \mathrm{NR}$, and there was no difference in CR rate between two groups (94\% vs 93\%; $p=.802)$.

\section{DLI for preventing relapse}

According to the DLI strategy mentioned above, a total of 199 patients received DLI for preventing relapse at a median time of 99 (range 60-640) days post-transplants, including 91 (76\%) cases in HID group and 108 (82\%) cases in MSD group ( $p=.350)$ (Fig. 1). Of them, 139 patients received prophylactic DLI (HID, $n=68$; MSD, $n=$ 71 ) and 72 preemptive DLI (HID, $n=28$; MSD, $n=44$ ). The median number of DLI was 1 (range $1-4$ ) per patient, with 1 (range 1-4) in each group, respectively ( $p=$ .087)). The median count of $\mathrm{CD}^{+} \mathrm{T}$ cells pDLI was 2.8 (range, $1.1-6.2$ ) $\times 10^{7} / \mathrm{kg}$ in HID group and 3.0 (range, $1.0-6.5) \times 10^{7} / \mathrm{kg}$ in MSD group, respectively $(p=.625)$. Until the last follow-up, 114 episodes of post-MRD+ were recorded in 92 patients including 60 cases before DLI and 12 after DLI. Of them, 18 patients experienced two, and 2 had three episodes of post-MRD+. The incidence of the first episode of post-MRD+ was 28\% (95\% CI, 24-32) and 45\% (95\% CI, 40-49; $p=.006)$ in HID and MSD groups, respectively.

\section{GVHD}

The 1-year cumulative incidences of grades II-IV aGVHD were $62 \%$ (95\% CI, 58-67) and 54\% (95\% CI, 50-58; $p=$ $.025)$, and III-IV aGVHD post-transplants were 16\% (95\% CI, $13-19)$ and $11 \%$ (95\% CI, 8-13; $p=.180)$ in HID and MSD groups, respectively. Of 199 patients received DLI, 70 (35\%) patients developed aGVHD, including 28 (31\%) in HID group and $42(39 \%)$ in MSD group $(p=.238)$. Among the 70 aGVHD, 48 (69\%) involved skin, 29 (41\%)

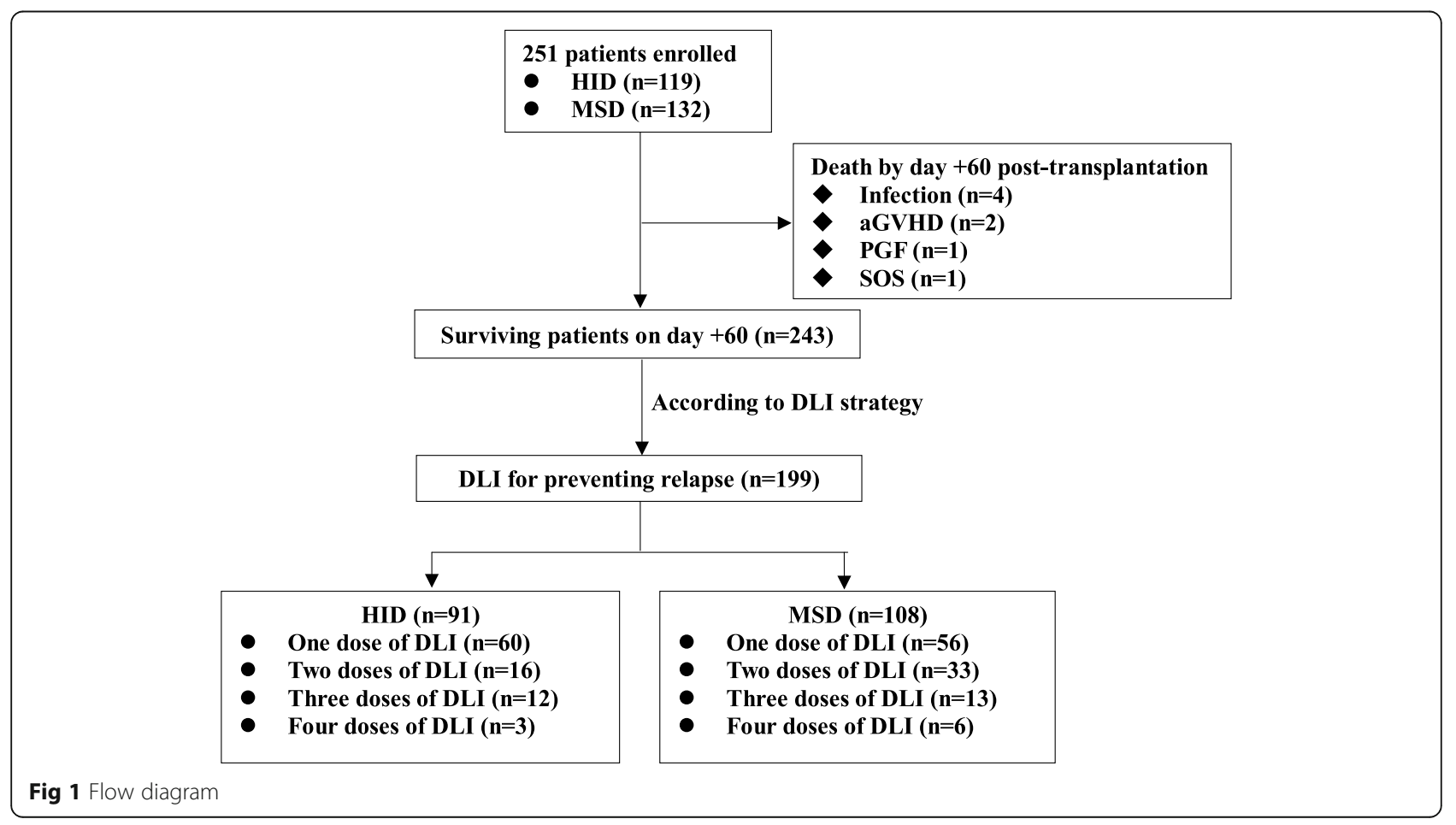


Table 1 Patients and transplant characteristics

\begin{tabular}{llll}
\hline Characteristics & HID & MSD & $p$ \\
\hline No. of patients & 119 & 132 & - \\
Median age, years (range) & $28(14-54)$ & $33(16-56)$ & .127 \\
Sex, no. (\%) & & & .448 \\
$\quad$ Male/female & $65(54.6) / 54$ & $65(49.2) / 67$ & \\
Underlying diseases, (\%) & $(45.4)$ & $(50.8)$ & \\
$\quad$ & & & .731 \\
AML & $54(45.4)$ & $57(43.2)$ & \\
ALL & $55(46.2)$ & $60(45.4)$ & \\
& $10(8.4)$ & $15(11.4)$ &
\end{tabular}

Disease stage at

transplants, (\%)

$\begin{array}{lll}\text { Primary induction failure } & 58(48.7) & 57(43.2) \\ \text { Relapse refractory } & 61(51.3) & 75(56.8)\end{array}$

WBC count at diagnosis, $n$

(\%)

$$
\begin{aligned}
& \geq 30,000 \text { per } \mathrm{mm}^{3} \\
& <30,000 \text { per } \mathrm{mm}^{3}
\end{aligned}
$$

Median BM blasts before conditioning (range)

\begin{tabular}{|c|c|c|c|}
\hline Poor & 65 (54.6) & $66(50.0)$ & \\
\hline Intermediate & 34 (28.6) & $45(34.1)$ & \\
\hline Favorable & $5(4.2)$ & $8(6.1)$ & \\
\hline Unknown & 15 (12.6) & $13(9.8)$ & \\
\hline Graft no. (\%) & & & - \\
\hline$B M+P B S C$ & $119(100)$ & $3(2.3)$ & \\
\hline PBSC & $0(0)$ & $129(97.7)$ & \\
\hline $\begin{array}{l}\text { Median MNCs, } 10^{8} / \mathrm{kg} \\
\text { (range) }\end{array}$ & $7.36(3.76-12.80)$ & $7.80(3.52-13.16)$ & .136 \\
\hline $\begin{array}{l}\text { MedianCD34 + count, } \\
10^{6} / \mathrm{kg} \text { (range) }\end{array}$ & $5.65(1.15-15.16)$ & $5.41(0.98-16.46)$ & .471 \\
\hline $\begin{array}{l}\text { Follow-up time in survivors } \\
\text { from transplant, median } \\
\text { (range), mouth }\end{array}$ & $43.4(20.5-71.5)$ & $41.6(18.2-72.7)$ & .814 \\
\hline
\end{tabular}

Genetics, (\%)

$A M L$ acute myelogenous leukemia, $A L L$ acute lymphoblastic leukemia, $A L A L$ acute leukemia of ambiguous lineage, $M N C$ mononuclear cell, $P B S C$ peripheral blood stem cell, $B M$ bone marrow

liver, and 24 (34\%) gastrointestinal tract. Eventually, 9 patients died of aGVHD in HID group and 5 in MSD group $(p$ $=.271)$. The 3-year cumulative incidences of cGVHD were $55 \%(95 \% \mathrm{CI}, 50-59)$ and 55\% (95\% CI, 51-60; $p=.789)$, and extensive cGHVD were $21 \%$ (95\% CI, 17-25) and 19\% (95\% CI, 16-22; $p=.830$ ) in HID and MSD groups, respectively. Seventy-seven (39\%) patients developed cGVHD after DLI, including 32 (35\%) in HID group and 45 (42\%) in MSD group ( $p=.383$ ). Totally, 18 cases were diagnosed with bronchiolitis obliterans, including 6 cases in HID and 12 in MSD groups respectively $(p=.232)$. Four patients died of cGVHD in HID group and 3 in MSD group ( $p=.711)$ (Table 3).
Risk factors for aGVHD included age, gender, donor type, and DLI. For cGVHD, the above variables as well as aGVHD were included. Multivariate analysis showed that HID and the number of DLI $\geq 2$ were risk factors for aGVHD $(p=.010, \mathrm{HR}=1.545 ; p=.029, \mathrm{HR}=1.463$, respectively). DLI was a risk factor for cGVHD in multivariate analysis $(p<.001, \mathrm{HR}=2.603)$.

\section{Relapse}

The 5-year cumulative incidence of relapse posttransplant for all patients was 32\% (95\% CI, 23-41), with $26 \%$ (95\% CI, 18-35) in HID group and 38\% (95\% CI, 29$47 ; p=.034$ ) in MSD group (Fig. 2a), respectively. The median time of relapse was 242 (range 62-1093) and 239 (range 46-1242) days post-transplantation $(p=.632)$ in HID and MSD groups, respectively.

Subgroup analysis revealed that 5-year cumulative incidence of relapse for AML,ALL, and ALAL was 23\% (95\% CI, 13-36), 27\% (95\% CI, 18-41), and 30\% (95\% CI, 6-60) in HID group, respectively, and 32\% (95\% CI, 20-45), 44\% (95\% CI, 29-58), and 40\% (95\% CI, 15-64) in MSD group, respectively (Fig. 2b for AML, $p=.243$; Fig. 2c for ALL, $p=.089$; Fig. 2d for ALAL, $p=.635$ ).

Of the 199 patients who received DLI, 56 relapsed, including 20 in HID and 36 in MSD groups, respectively. Of 127 patients received prophylactic DLI, 32 (25\%) relapsed. Of the 60 patients received preemptive DLI, 19 (32\%) relapsed. Of the 12 patients received both prophylactic and preemptive DLI, $5(42 \%)$ relapsed. In DLI subgroup $(n=199)$, the cumulative incidence of relapse was 30\% (24\% in HID and 35\% in MSD groups, respectively $(p=.065))$. In non-DLI subgroup $(n$ $=52$ ), 21 cases relapsed. Eight post-MRD+ cases with active GVHD received preemptive treatment of decitabine, and 4 patients relapsed. The cumulative incidence of relapse of this subgroup was $43 \%$ (33\% in HID and 57\% in MSD groups, respectively $(p=.107))$.

Of the 77 relapsed patients, 27 received DLI plus chemotherapy, 20 chemotherapy alone, 4 seconds allo-HSCT, and 26 abandoned further therapy. Of the 51 patients underwent treatments, 18 achieved the second CR, and 7 are still alive. Univariate analysis showed that relapse refractory, the percentage of BM blasts $\geq 3 \%$ on day 0 and post-MRD+, was an adverse factor for relapse $(p=.048, \mathrm{HR}=1.448 ; p=$ $.004, \mathrm{HR}=1.941 ; p=.002$, HR $=1.996$, respectively). In multivariate analysis, the percentage of BM blasts $\geq 3 \%$ on day 0 and post-MRD+ was an adverse factor for relapse ( $p$ $=.037, \mathrm{HR}=1.652 ; p=.003, \mathrm{HR}=2.019$, respectively). HID, DLI, and cGVHD were protective factors for relapse $(p=.047, \mathrm{HR}=0.615 ; p=.034, \mathrm{HR}=0.561 ; p=.023, \mathrm{HR}=$ 0.580 , respectively) (Table 2 ).

\section{Infections}

In total, 41 patients died of infections, including 22 in HID group and 19 in MSD group, and the overall 


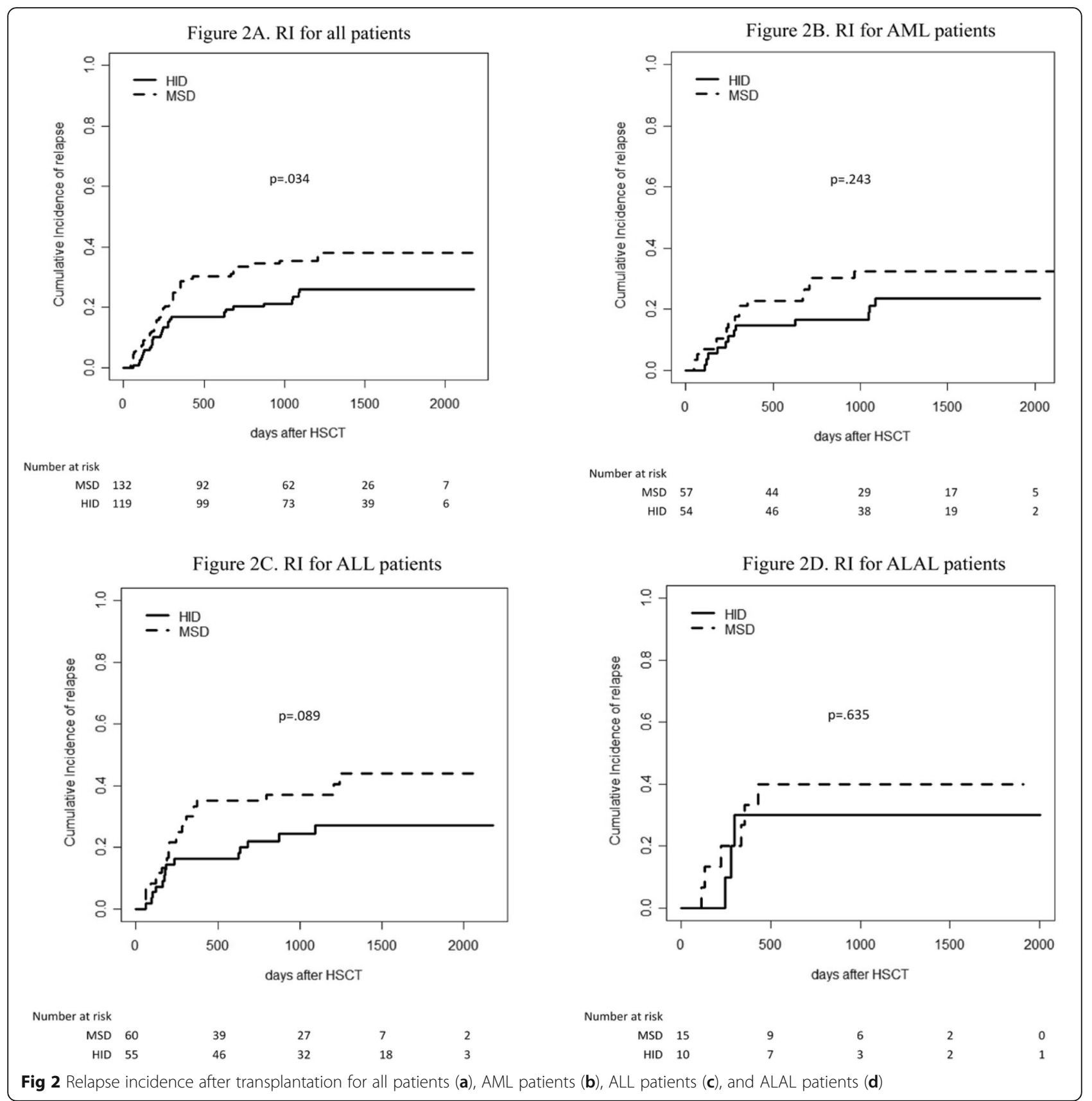

incidences of infection-related mortality were 19\% (95\% CI, 15-22) and $14 \%$ (95\% CI,11-18), respectively, with no difference between two groups $(p=.352)$. However, within the first 100 days' post-transplant, there was higher infection-related mortality in HID than MSD $(8 \%$ vs $2 \%, p=.049)$.

The 1-year cumulative incidence of EBV-emia was $46 \%$ (95\% CI, $42-51)$ and $17 \%$ (95\% CI, 14-21; $p<.001)$ in HID and MSD groups, respectively. The 2-year cumulative incidence of EBV-associated diseases was 14\% (95\% CI, 11-18) and $6 \%(95 \% \mathrm{CI}, 4-8 ; p=.027)$ in two groups, respectively. Ten died of EBV-associated diseases, including 6 in HID group and 4 cases in MSD group (5\% vs 3\%; $p=.524$ ). The 1-year cumulative incidence of CMV-emia was $66 \%$ (95\% CI, $62-71)$ and $41 \%(95 \% \mathrm{CI}, 37-45 ; p<.001)$ in two groups, respectively. The 2-year cumulative incidence of CMVassociated diseases was 6\% (95\% CI, 4-8) and 6\% (95\% CI, $4-8 ; p=.977)$ in two groups, respectively. Five died of CMV-associated diseases, including 3 in HID group and 2 cases in MSD group (3\% vs $2 \% ; p=.670$ ).

\section{Survival}

At a median follow-up of 20.3 (range, 0.2-72.7) months post-transplantation, 114 patients survived, and 137 died 
Table 2 Univariate and multivariate analysis for relapse, DFS, and OS

\begin{tabular}{|c|c|c|c|c|c|c|}
\hline \multirow[t]{2}{*}{ Variable } & \multicolumn{2}{|l|}{ Relapse } & \multicolumn{2}{|l|}{ DFS } & \multicolumn{2}{|l|}{ OS } \\
\hline & Univariate & Multivariate (HR) & Univariate & Multivariate (HR) & Univariate & Multivariate (HR) \\
\hline HID vs MSD & $P=.034$ & $\begin{array}{l}P=.047(0.615) \\
95 \% \mathrm{Cl} 0.381-0.994\end{array}$ & $P=.665$ & - & $P=.832$ & - \\
\hline Male/female & $P=.362$ & - & $P=.680$ & - & $P=.433$ & - \\
\hline $\begin{array}{l}\text { Patient age, } \geq 28 \text { years } \\
\text { old vs }<28 \text { years old }\end{array}$ & $P=.183$ & - & $P=.219$ & - & $P=.376$ & - \\
\hline AML/non-AML & $P=.204$ & - & $P=.518$ & - & $P=.448$ & - \\
\hline $\begin{array}{l}\text { Genetics:poor-risk vs } \\
\text { others }\end{array}$ & $P=.321$ & - & $P=.254$ & - & $P=.489$ & - \\
\hline $\begin{array}{l}\text { Disease stage at } \\
\text { transplants:relapse } \\
\text { refractory vs PIF }\end{array}$ & $P=.048$ & $\begin{array}{l}P=.121(1.449) \\
95 \% \mathrm{Cl} 0.907-2.314\end{array}$ & $P=.084$ & $\begin{array}{l}P=.148(1.288) \\
95 \% \mathrm{Cl} 0.914-1.815\end{array}$ & $P=.131$ & - \\
\hline $\begin{array}{l}\text { WBC count at } \\
\text { diagnosis:high } \\
\text { vs others }\end{array}$ & $P=.659$ & - & $P=.343$ & - & $P=.247$ & - \\
\hline $\begin{array}{l}\text { BM blasts before } \\
\text { conditioning, } \geq \\
32 \% \text { vs }<32 \% \\
\text { (median) }\end{array}$ & $P=.191$ & - & $P=.206$ & - & $P=.245$ & - \\
\hline $\begin{array}{l}\text { BM blasts on day } \\
0, \geq 3 \% \text { vs }<3 \% \\
\text { (median) }\end{array}$ & $P=.004$ & $\begin{array}{l}P=.037(1.652) \\
95 \% \mathrm{Cl} 1.032-2.640\end{array}$ & $P<.001$ & $\begin{array}{l}P=.005(1.630) \\
95 \% \mathrm{Cl} 1.162-2.288\end{array}$ & $P=.001$ & $\begin{array}{l}P=.011(1.573) \\
95 \% C l 1.110-2.229\end{array}$ \\
\hline $\begin{array}{l}\text { MRD status } \\
\text { post-transplant, } \\
\text { pos vs neg }\end{array}$ & $P=.002$ & $\begin{array}{l}\mathrm{P}=.003(2.019) \\
95 \% \mathrm{Cl} 1.267-3.216\end{array}$ & $P=.005$ & $\begin{array}{l}P=.003(1.668) \\
95 \% \mathrm{Cl} 1.185-2.347\end{array}$ & $P=.019$ & $\begin{array}{c}P=.027(1.490) \\
95 \% \mathrm{Cl} 1.048-2.121\end{array}$ \\
\hline DLI vs no DLI & $P=.025$ & $\begin{array}{l}P=.034(0.561) \\
95 \% \mathrm{Cl} 0.329-0.957\end{array}$ & $P<.001$ & $\begin{array}{l}P<.001(0.402) \\
95 \% \mathrm{Cl} 0.275-0.588\end{array}$ & $P<.001$ & $\begin{array}{l}P<.001(0.423) \\
95 \% \mathrm{Cl} 0.289-0.620\end{array}$ \\
\hline $\begin{array}{l}\text { II-IV aGVHD Vs } \\
0-I \text { aGVHD }\end{array}$ & $P=.212$ & - & $P=.530$ & - & $P=.558$ & - \\
\hline $\begin{array}{l}\text { cGVHD vs no } \\
\text { cGVHD }\end{array}$ & $P=.003$ & $\begin{array}{l}P=.023(0.580) \\
95 \% \mathrm{Cl} 0.363-0.928\end{array}$ & $P=.003$ & $\begin{array}{l}P=.016(0.659) \\
95 \% \mathrm{Cl} 0.470-0.925\end{array}$ & $P=.050$ & $\begin{array}{l}P=.108(0.788) \\
95 \% \mathrm{Cl} 0.557-1.116\end{array}$ \\
\hline
\end{tabular}

HID haploidentical related donor, $M S D$ matched sibling donor, $A M L$ acute myelogenous leukemia, non- $A M L$ acute leukemia other than AML, OS overall survival, DFS disease free survival, PIF primary induction failure, BM bone marrow, high WBC count at diagnosis WBC count $\geq 30,000$ per mm ${ }^{3}$, MRD minimal residual disease

(63 in HID and 74 in MSD). The causes of death included relapse $(n=69)$, infectious diseases $(n=$ $41)$, GVHD $(n=21)$, sinusoidal obstruction syndrome $(n=1)$, thrombotic microangiopathy $(n=1)$, multiple organs failure $(n=2)$, primary graft failure $(n=1)$, and unknown $(n=1)$ (Table 3$)$. The 5-year cumulative incidence of NRM was $31 \%$ (95\% CI, 22-40) and 23\% (95\% CI,16-30; $p=.114$ ) (Fig. 3a) in HID and MSD groups, respectively. The 5-year OS was $46 \%(95 \% \mathrm{CI}, 42-51)$ and $42 \%$ (95\% CI,3746 ; $p=.832$ ) (Fig. 3b), DFS was $43 \%$ (95\% CI, 3848 ) and $39 \%(95 \% \mathrm{CI}, 35-44 ; p=.665)$ (Fig. 3c), and GRFS was $28 \%(95 \% \mathrm{CI}, 24-33)$ and $26 \%$ (95\% CI, 22-30; $p=.795$ ) (Fig. 3d) in HID and MSD groups, respectively.

Subgroup analysis showed that 5-year OS for AML, ALL, and ALAL was 50\% (95\% CI, 43-57), $43 \%$ (95\% CI, 36-51), and 40\% (95\% CI, 27-53) in HID group, respectively, and 44\% (95\% CI, 38-51),
$38 \%(95 \% \mathrm{CI}, 31-46)$, and $40 \%$ (95\% CI, 25-56) in MSD group, respectively, and there was no difference between two groups for each underlying disease $(p=.947$ for AML; $p=.798$ for ALL; $p=.927$ for ALAL).

Multivariate analysis showed that DLI was a protective factor for OS and DFS $(p<.001, \mathrm{HR}=$ $0.423 ; p<.001, \mathrm{HR}=0.402$, respectively), and cGVHD was associated with better DFS $(p=.016$,

Table 3 Causes of death post-transplantation

\begin{tabular}{llll}
\hline $\begin{array}{l}\text { Cause of } \\
\text { death }\end{array}$ & HID group & MSD group & $p$ \\
\hline nelapse & $26(21.8)$ & $43(32.6)$ & .057 \\
aGVHD & $9(7.6)$ & $5(3.8)$ & .271 \\
cGVHD & $4(5.9)$ & $3(3.7)$ & .711 \\
Infections & $22(18.5)$ & $19(14.4)$ & .398 \\
others & $2(1.7)$ & $4(3.0)$ & .686 \\
\hline
\end{tabular}


A
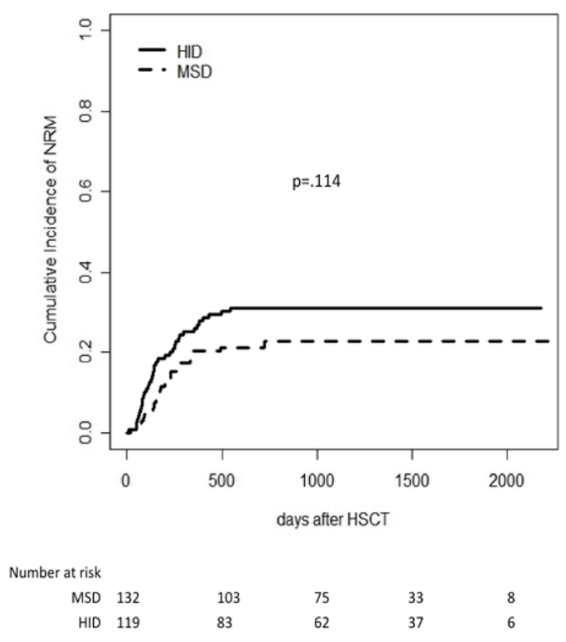

$\mathrm{C}$

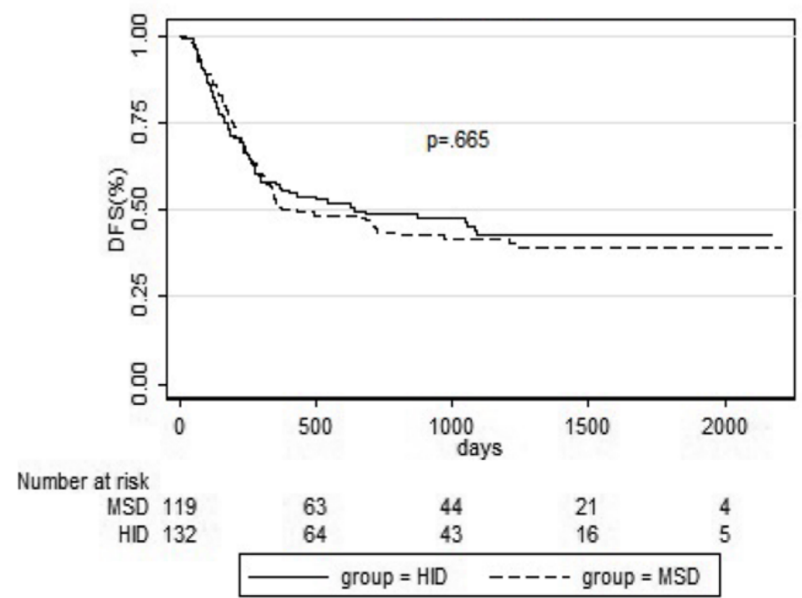

B

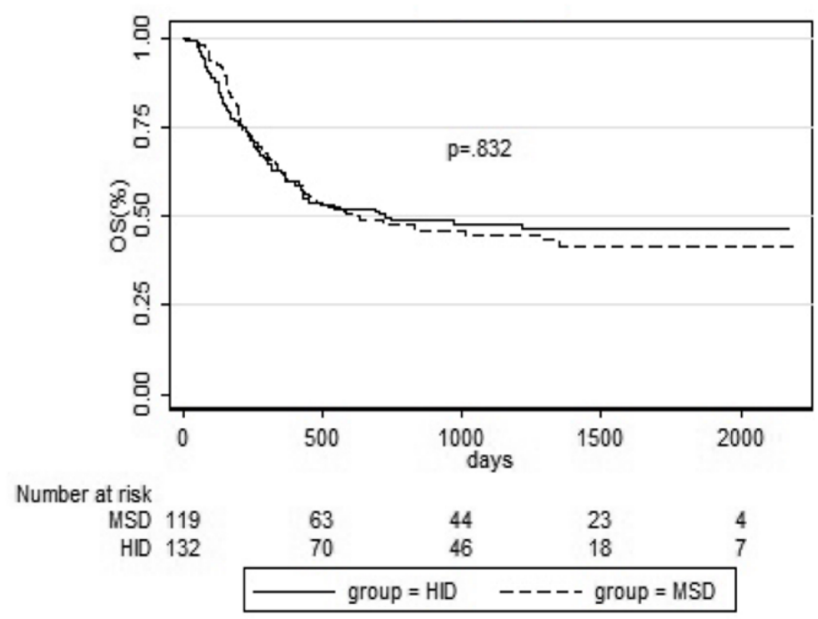

D

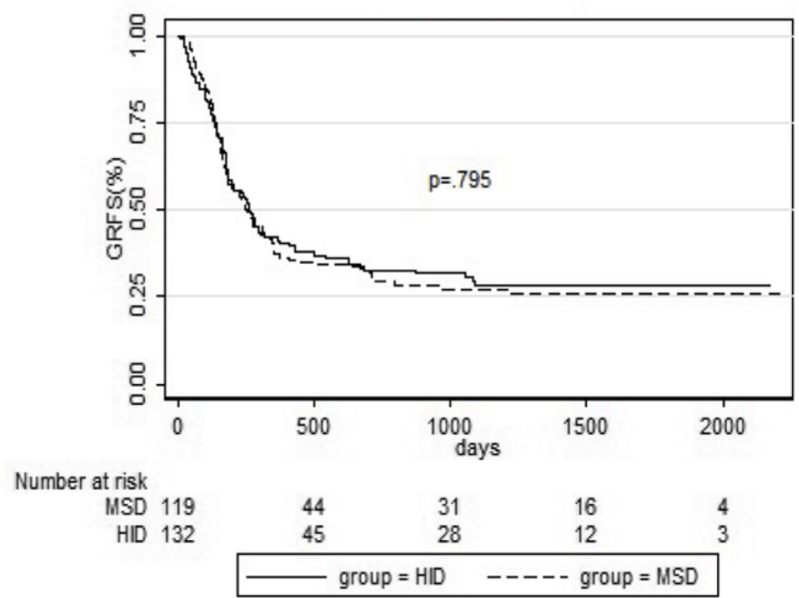

Fig 3 Transplant outcomes including $\operatorname{NRM}(\mathbf{a})$, OS (b), DFS (c), and GRFS (d)

$\mathrm{HR}=0.659)$. The percentage of $\mathrm{BM}$ blasts $\geq 3 \%$ on day 0 and post-MRD+ was an adverse factor for OS $(p=.011, \mathrm{HR}=1.573 ; p=.027, \mathrm{HR}=1.490$, respectively) and DFS $(p=.005$, HR $=1.630 ; p=$ $.003, \mathrm{HR}=1.668$, respectively) (Table 2$)$.

\section{Discussion}

For the patients with refractory acute leukemia, allo-HSCT is the optimal curative treatment, which may allow $17-53 \%$ of patients to achieve long-term survival [7, 9, 10, 34]. Fascinatingly, a promising strategy of salvage chemotherapy with sequential conditioning was reported by our and other transplant centers, with a chance of $30-53 \% 3$-year or 5year OS $[7,10,35,36]$. In this study, we obtained similar survival results $[7,10]$. For patients lacking an MSD or suitably unrelated donor, HID can be an alternative choice because of its rapid and easy availability. A recent large sample data from EBMT registry [16] revealed that HID transplant had similar RI, higher NRM, and inferior survival rates than MSD transplant for refractory/relapse acute leukemia. Compared with the study of EBMT, our result showed that HID transplant had lower RI compared with MSD transplant in refractory acute leukemia. The result was consistent with our historical studies $[18,21]$ and other studies [17, 19], which had lower RI in HID for highrisk leukemia. In this study, our results showed that HID transplant also had a higher incidence of NRM compared with MSD, but it did not show a statistical difference between two groups. Though there was significantly lower RI in HID than MSD, the incidences of OS and DFS was similar between two groups. The reasonable explanation for these results might be the advantage of lower RI in HID group that was offset by relatively higher NRM.

Relapse is a major cause of treatment failure after alloHSCT, especially for refractory leukemia. Many factors might influence relapse, such as transplant strategy, underlying diseases, donor resources, genetics, and disease status at transplantation $[7,16,22,37]$. The data of 
EBMT showed that 2-year relapse rates for refractory/relapse leukemia were $50 \%$ in haplotransplant and $51 \%$ in MSD transplant, respectively [16]. Our result showed that the 5-year cumulative incidence of relapse was 32\% in this population of refractory acute leukemia, with $25 \%$ in HID and 38\% in MSD transplants. Compared with outcomes of EBMT, ours were better regardless of HID or MSD. The favorable outcomes might be attributed to the following two aspects: sequential intensified conditioning greatly decreased the leukemia burden and early tapering of immunosuppressant combined with DLI further enhanced anti-leukemia effect [7, 10, 35, 38]. Some studies suggested that AML had lower RI and better OS than ALL for refractory or advanced-stage diseases [39-41] and this study as well as our previous report [10] showed that AML and ALL had comparable RI and OS, suggesting the strategy of sequential intensified conditioning followed by DLI might be equally beneficial for both diseases. For donor resources, some studies showed that HID had stronger GVL than MSD, making relapse lower [17-19, 22, 42]. Conversely, some other studies found no advantage of GVL in haplo setting $[13,16,43]$. Our results showed that HID was associated with lower incidence of post-MRD+ and hematologic relapse compared with MSD and proved to be a protective factor in multivariate analysis of relapse, suggesting a superior GVL for refractory acute leukemia. In subgroup analysis, HID had a lower relapse tendency than MSD for ALL $(p=.089)$. Poor genetics was not a related factor to relapse, which might be explained by that all patients enrolled in this study were diagnosed with refractory leukemia, and most of them were poor-risk in genetics. Higher percentage of $\mathrm{BM}$ blasts on day 0 posttransplantation was an adverse factor for relapse, as we reported previously [10]. Besides, Schmid et al. observed that it is more effective for PIF than relapse refractory patients by using the strategy of sequential conditioning followed by DLI [35, 38].Our results showed that patients with relapse refractory diseases had a higher risk of relapse than PIF in univariate analysis, but not in multivariate analysis.

Two main causes of NRM included GVHD and infections. In this study, a T cell replete protocol by using ATG for GVHD prophylaxis was implemented in HID transplant. Our results showed that after excluding the effect of DLI, grades II-IV aGVHD were higher for HID than MSD, but severe aGVHD and cGVHD were comparable between two groups, consistent with our data previously [22, 44, 45]. In order to induce GVL, a strategy of Immunosupressant withdrawal and DLI was used. The two methods both face the risk of GVHD. In this study, although the incidences of GVHD increased in both HID and MSD settings, the mortality of GVHD was acceptable, which was attributed to the effective interventions of DLI-related GVHD [10, 39, 46]. Meanwhile, there was no difference in terms of both aGVHD and cGVHD between two groups, consistent with our historical reports [44, 45].
Infection-related mortality was a major barrier to success of transplant. Some studies found that sequential intensified conditioning and ATG were associated with high incidence of infections, especially in the early period posttransplantation [24, 25, 47, 48]. Recently, our multicenter randomized study found that sequential intensified conditioning along with ATG for GVHD prophylaxis was positively associated with viral infections [24]. In the current study, despite no difference in overall infection-related mortality, HID transplant had higher infection-related deaths than MSD transplant within 100 day posttransplantation, coherent with our previous result [24].

The major limitation of this study was that it is a retrospective analysis of two prospective data. Therefore, further prospective multicenter studies are needed to validate our findings.

\section{Conclusions}

In conclusion, HID transplant has lower relapse, but higher infection-related mortality and similar survival rates in refractory acute leukemia by the strategy of sequential intensified conditioning followed by DLI compared with MSD transplant.

Acknowledgements
We thank all the faculty members that participated in this study.

\section{Authors'contributions}

QFL and YW designed the study. SJY, FH, and ZPF analyzed the data and drafted the manuscript. All authors contributed to the data collection, data interpretation, manuscript preparation, and approval of the final version.

\section{Funding}

This work was supported by the National Key Research and Development Programme of China (Grant No. 2017YFA105500, 2017YFA105504),the National Natural Science Foundation of China (No. 81770190, 81700176, 81470349,81770189), the Clinical Research Nurture Project of Nanfang Hospital, Southern Medical University (No. LC2016PY018, LC2016ZD009), the Clinical Research Project of Nanfang Hospital, and Southern Medical University (No. 2018CR044).

\section{Availability of data and materials}

All data generated or analyzed during this study are included in this published article.

\section{Competing interest}

The authors declare that they have no competing interests.

\section{Ethics approval and consent to participate}

The study was performed in accordance with the modified Helsinki Declaration, and the protocol was approved by our ethical review boards before study initiation. Informed consent was obtained from the donors and recipients.

\section{Consent for publication \\ Not applicable.}

\section{Author details}

'Department of Hematology, Nanfang Hospital, Southern Medical University, No.1838 North Guangzhou Avenue, Guangzhou 510515, China. ${ }^{2}$ Department of Hematology, Peking University People's Hospital, Peking University Institute of Hematology, No.11 South Street of Xizhimen, Xicheng District, Beijing 100044, China. ' ${ }^{3}$ Department of Hematology, Sun Yat-Sen Memorial Hospital, Sun Yat-Sen University, No.107 Yanjiang West Road, Guangzhou 510120, China. ${ }^{4}$ Department of Hematology, Xiangya Hospital, Central South 
University, No.87 Xiangya Road, Changsha 410008, China. ${ }^{5}$ Department of Hematology, Fujian Institute of Hematology, Fujian Medical University Union Hospital, No.29 Xinquan Road, Fuzhou 350001, China. ${ }^{6}$ Department of Hematology, Guangzhou First People's Hospital, No.1 Fupan Road, Guangzhou 510180, China. 'Department of Hematology, Guangzhou General Hospital of Guangzhou Military Command, No.111 Liuhua Road, Guangzhou 510010, China. ${ }^{8}$ Department of Hematology, the Third Affiliated Hospital, Sun Yat-Sen University, No.600 Tianhe Road, Guangzhou 510000, China. ${ }^{9}$ Department of Hematology, Nanhai Hospital, Southern Medical University, Foshan, China.

\section{Received: 15 November 2019 Accepted: 5 March 2020}

\section{-}

\section{References}

1. Gyurkocza B, Lazarus HM, Giralt S. Allogeneic hematopoietic cell transplantation in patients with AML not achieving remission: potentially curative therapy. Bone Marrow Transplant. 2017;52:1083-90.

2. Biggs JC, Horowitz MM, Gale RP, et al. Bone marrow transplants may cure patients with acute leukemia never achieving remission with chemotherapy. Blood. 1992;80:1090-3.

3. Middeke JM, Herbst R, Parmentier S, et al. Long-term follow-up and impact of comorbidity before allogeneic hematopoietic stem cell transplantation in patients with relapsed or refractory acute myeloid leukemia-lessons learned from the prospective BRIDGE trial. Biol Blood Marrow Transplant. 2017;23:1491-7.

4. $\mathrm{Xu} \mathrm{L}$, Chen $\mathrm{H}$, Chen J, et al. The consensus on indications, conditioning regimen, and donor selection of allogeneic hematopoietic cell transplantation for hematological diseases in China-recommendations from the Chinese Society of Hematology. J Hematol Oncol. 2018;11:33.

5. Saraceni F, Labopin M, Brecht A, et al. Fludarabine-treosulfan compared to thiotepa-busulfan-fludarabine or FLAMSA as conditioning regimen for patients with primary refractory or relapsed acute myeloid leukemia: a study from the Acute Leukemia Working Party of the European Society for Blood and Marrow Transplantation (EBMT). J Hematol Oncol. 2019;12:44.

6. Steckel NK, Groth C, Mikesch JH, et al. High-dose melphalan-based sequential conditioning chemotherapy followed by allogeneic haematopoietic stem cell transplantation in adult patients with relapsed or refractory acute myeloid leukaemia. Br J Haematol. 2018;180:840-53.

7. Liu QF, Fan ZP, Zhang Y, et al. Sequential intensified conditioning and tapering of prophylactic immunosuppressants for graft-versus-host disease in allogeneic hematopoietic stem cell transplantation for refractory leukemia. Biol Blood Marrow Transplant. 2009;15:1376-85.

8. Malard F, Labopin M, Stuhler G, et al. Sequential intensified conditioning regimen allogeneic hematopoietic stem cell transplantation in adult patients with intermediate- or high-risk acute myeloid leukemia in complete remission: a study from the Acute Leukemia Working Party of the European Group for Blood and Marrow Transplantation. Biol Blood Marrow Transplant. 2017:23:278-84.

9. Mohty M, Malard F, Blaise D, et al. Sequential regimen of clofarabine, cytosine arabinoside and reduced-intensity conditioned transplantation for primary refractory acute myeloid leukemia. Haematologica. 2017;102:184-91.

10. Xuan $L$, Fan Z, Zhang Y, et al. Sequential intensified conditioning followed by prophylactic DLI could reduce relapse of refractory acute leukemia after allo-HSCT. Oncotarget. 2016;7:32579-91.

11. Versluis J, Labopin M, Ruggeri A, et al. Alternative donors for allogeneic hematopoietic stem cell transplantation in poor-risk AML in CR1. Blood Adv. 2017;: :477-85

12. Wang $Y$, Liu $Q F$, Xu LP, et al. Haploidentical vs identical-sibling transplant for AML in remission: a multicenter, prospective study. Blood. 2015;125:3956-62.

13. Bashey A, Zhang X, Sizemore CA, et al. T-cell-replete HLA-haploidentical hematopoietic transplantation for hematologic malignancies using posttransplantation cyclophosphamide results in outcomes equivalent to those of contemporaneous HLA-matched related and unrelated donor transplantation. J Clin Oncol. 2013;31:1310-6.

14. Rashidi A, Hamadani M, Zhang MJ, et al. Outcomes of haploidentical vs matched sibling transplantation for acute myeloid leukemia in first complete remission. Blood Adv. 2019;3:1826-36.

15. How J, Slade M, Vu K, et al. T cell-replete peripheral blood haploidentical hematopoietic cell transplantation with post-transplantation cyclophosphamide results in outcomes similar to transplantation from traditionally matched donors in active disease acute myeloid leukemia. Biol Blood Marrow Transplant. 2017;23:648-53.

16. Battipaglia G, Boumendil A, Labopin M, et al. Unmanipulated haploidentical versus $H L A$-matched sibling allogeneic hematopoietic stem cell transplantation in relapsed/refractory acute myeloid leukemia: a retrospective study on behalf of the ALWP of the EBMT. Bone Marrow Transplant. 2019.

17. Ottinger HD, Ferencik S, Beelen DW, et al. Hematopoietic stem cell transplantation: contrasting the outcome of transplantations from HLAidentical siblings, partially HLA-mismatched related donors, and HLAmatched unrelated donors. Blood. 2003;102:1131-7.

18. Wang $Y$, Liu $D H$, Xu LP, et al. Superior graft-versus-leukemia effect associated with transplantation of haploidentical compared with HLAidentical sibling donor grafts for high-risk acute leukemia: an historic comparison. Biol Blood Marrow Transplant. 2011;17:821-30.

19. Luo Y, Xiao H, Lai X, et al. T-cell-replete haploidentical HSCT with low-dose anti-T-lymphocyte globulin compared with matched sibling HSCT and unrelated HSCT. Blood. 2014;124:2735-43.

20. Zhao XS, Liu YR, Xu LP, et al. Minimal residual disease status determined by multiparametric flow cytometry pretransplantation predicts the outcome of patients with ALL receiving unmanipulated haploidentical allografts. Am J Hematol. 2019;94:512-21.

21. Yu S, Huang F, Wang Y, et al. Haploidentical transplantation might have superior graft-versus-leukemia effect than HLA-matched sibling transplantation for high-risk acute myeloid leukemia in first complete remission: a prospective multicentre cohort study. Leukemia. 2019.

22. Chang YJ, Wang Y, Liu YR, et al. Haploidentical allograft is superior to matched sibling donor allograft in eradicating pre-transplantation minimal residual disease of AML patients as determined by multiparameter flow cytometry: a retrospective and prospective analysis. J Hematol Oncol. 2017;10:134.

23. Wang $Y$, Liu DH, Xu LP, et al. Prevention of relapse using granulocyte CSFprimed PBPCs following HLA-mismatched/haploidentical, T-cell-replete hematopoietic SCT in patients with advanced-stage acute leukemia: a retrospective risk-factor analysis. Bone Marrow Transplant. 2012;47:1099-104.

24. Lin R, Wang Y, Huang F, et al. Two dose levels of rabbit antithymocyte globulin as graft-versus-host disease prophylaxis in haploidentical stem cell transplantation: a multicenter randomized study. BMC Med. 2019;17:156.

25. Xuan L, Huang F, Fan Z, et al. Effects of intensified conditioning on Epstein-Barr virus and cytomegalovirus infections in allogeneic hematopoietic stem cell transplantation for hematological malignancies. J Hematol Oncol. 2012;5:46.

26. Liu Q, Lin R, Sun J, et al. Antifungal agents for secondary prophylaxis based on response to initial antifungal therapy in allogeneic hematopoietic stem cell transplant recipients with prior pulmonary aspergillosis. Biol Blood Marrow Transplant. 2014;20:1198-203.

27. Holtan SG, DeFor TE, Lazaryan A, et al. Composite end point of graft-versushost disease-free, relapse-free survival after allogeneic hematopoietic cell transplantation. Blood. 2015;125:1333-8.

28. Przepiorka D, Weisdorf D, Martin P, et al. 1994 consensus conference on acute GVHD grading. Bone Marrow Transplant. 1995;15:825-8.

29. Horwitz ME, Sullivan KM. Chronic graft-versus-host disease. Blood Rev. 2006;20:15-27.

30. Arber DA, Orazi A, Hasserjian R, et al. The 2016 revision to the World Health Organization classification of myeloid neoplasms and acute leukemia. Blood. 2016;127:2391-405.

31. O'Donnell MR, Tallman MS, Abboud CN, et al. Acute myeloid leukemia, version 3.2017, NCCN clinical practice guidelines in oncology. J Natl Compr Canc Netw. 2017:15:926-57.

32. Alvarnas JC, Brown PA, Aoun P et al. Acutellymphoblastic Leukemia, Version 2.2015. J Natl Compr Canc Netw 2015; 13: 1240-1279.

33. Austin PC, Fine JP. Practical recommendations for reporting Fine-Gray model analyses for competing risk data. Stat Med. 2017;36:4391-400.

34. Ruggeri A, Battipaglia G, Labopin M, et al. Unrelated donor versus matched sibling donor in adults with acute myeloid leukemia in first relapse: an ALWP-EBMT study. J Hematol Oncol. 2016;9:89.

35. Schmid C, Schleuning M, Schwerdtfeger R, et al. Long-term survival in refractory acute myeloid leukemia after sequential treatment with chemotherapy and reduced-intensity conditioning for allogeneic stem cell transplantation. Blood. 2006;108:1092-9.

36. Ringden $\mathrm{O}$, Labopin $\mathrm{M}$, Schmid $\mathrm{C}$, et al. Sequential chemotherapy followed by reduced-intensity conditioning and allogeneic haematopoietic stem cell transplantation in adult patients with relapse or refractory acute myeloid leukaemia: a survey from the Acute Leukaemia Working Party of EBMT. Br J Haematol. 2017;176:431-9. 
37. Wu X, Liu Q. Prophylaxis and treatment of relapse after haploidentical stem cell transplantation: What is known vs unknown? Semin Hematol. 2019:56:209-14.

38. Schmid C, Schleuning M, Ledderose G, et al. Sequential regimen of chemotherapy, reduced-intensity conditioning for allogeneic stem-cell transplantation, and prophylactic donor lymphocyte transfusion in high-risk acute myeloid leukemia and myelodysplastic syndrome. J Clin Oncol. 2005;23:5675-87.

39. Wang Y, Liu DH, Fan ZP, et al. Prevention of relapse using DLI can increase survival following HLA-identical transplantation in patients with advancedstage acute leukemia: a multi-center study. Clin Transplant. 2012;26:635-43.

40. Michallet $M$, Thomas $X$, Vernant JP, et al. Long-term outcome after allogeneic hematopoietic stem cell transplantation for advanced stage acute myeloblastic leukemia: a retrospective study of 379 patients reported to the Societe Francaise de Greffe de Moelle (SFGM). Bone Marrow Transplant. 2000;26:1157-63.

41. Doney $\mathrm{K}$, Hagglund $\mathrm{H}$, Leisenring $\mathrm{W}$, et al. Predictive factors for outcome of allogeneic hematopoietic cell transplantation for adult acute lymphoblastic leukemia. Biol Blood Marrow Transplant. 2003;9:472-81.

42. Salvatore D, Labopin M, Ruggeri A, et al. Outcomes of hematopoietic stem cell transplantation from unmanipulated haploidentical versus matched sibling donor in patients with acute myeloid leukemia in first complete remission with intermediate or high-risk cytogenetics: a study from the Acute Leukemia Working Party of the European Society for Blood and Marrow Transplantation. Haematologica. 2018;103:1317-28.

43. Ringden $\mathrm{O}$, Labopin $\mathrm{M}$, Ciceri $\mathrm{F}$, et al. Is there a stronger graft-versusleukemia effect using HLA-haploidentical donors compared with HLAidentical siblings? Leukemia. 2016;30:447-55.

44. Han $\sqcup$, Wang Y, Fan ZP, et al. Haploidentical transplantation compared with matched sibling and unrelated donor transplantation for adults with standard-risk acute lymphoblastic leukaemia in first complete remission. Br J Haematol. 2017;179:120-30.

45. Yu S, Fan Q, Sun J, et al. Haploidentical transplantation without in vitro Tcell depletion results in outcomes equivalent to those of contemporaneous matched sibling and unrelated donor transplantation for acute leukemia. Medicine (Baltimore). 2016;95:e2973.

46. Yan CH, Liu DH, Liu KY, et al. Risk stratification-directed donor lymphocyte infusion could reduce relapse of standard-risk acute leukemia patients after allogeneic hematopoietic stem cell transplantation. Blood. 2012:119:3256-62.

47. Binkert L, Medinger M, Halter JP, et al. Lower dose anti-thymocyte globulin for GvHD prophylaxis results in improved survival after allogeneic stem cell transplantation. Bone Marrow Transplant. 2015;50:1331-6.

48. Lin R, Liu Q. Diagnosis and treatment of viral diseases in recipients of allogeneic hematopoietic stem cell transplantation. J Hematol Oncol. 2013;6:94.

\section{Publisher's Note}

Springer Nature remains neutral with regard to jurisdictional claims in published maps and institutional affiliations.

Ready to submit your research? Choose BMC and benefit from:

- fast, convenient online submission

- thorough peer review by experienced researchers in your field

- rapid publication on acceptance

- support for research data, including large and complex data types

- gold Open Access which fosters wider collaboration and increased citations

- maximum visibility for your research: over $100 \mathrm{M}$ website views per year

At $\mathrm{BMC}$, research is always in progress.

Learn more biomedcentral.com/submissions 